\title{
Editorial over the Many Faces of Vitamin D in Chronic Kidney Disease: from Mineral to Immune-Inflammatory Modulator
}

\author{
Patrick M. Honore ${ }^{1,2}$ and Herbert D. Spapen ${ }^{1}$
}

Vitamin D (vit D), whether produced in the skin or absorbed from the diet, is first metabolized in the liver to generate 25-hydroxyvitamin D (25OHD) and then hydroxylated in the proximal renal tubule to 1,25 dihydroxyvitamin $\mathrm{D}\left(1,25(\mathrm{OH})_{2} \mathrm{D}\right) \cdot 1,25(\mathrm{OH})_{2} \mathrm{D}$ (calcitriol) is the major biologically active metabolite and serves a paracrine/autocrine function. $1,25(\mathrm{OH})_{2} \mathrm{D}$ forms a complex with the vit $\mathrm{D}$ nuclear receptor which binds to vit D response elements in the deoxyribonucleic acid (DNA). Thousands of these binding sites regulate hundreds of genes creating a multitude of genomic effects that modulate cell activation, proliferation, and differentiation within the immune-inflammatory system [1]. This underpins the biological rationale for a potential beneficial partnership of vit $\mathrm{D}$ in a variety of immune-inflammatory conditions that largely transcends its undisputed regulatory role in calcium/phosphate homeostasis and bone mineralization $[2,3]$.

Chronic kidney disease (CKD) is an intricate pathology characterized by a state of accelerated cardiovascular deterioration. Persistent inflammation has been recognized as an important component of CKD and may in part account for cardiovascular and all-cause mortality [4]. Low levels of both $25 \mathrm{OHD}$ and $1,25(\mathrm{OH})_{2} \mathrm{D}$ are observed in patients with CKD and are associated with higher mortality and faster progression of kidney disease [5]. In a recent issue of Inflammation, Zhao et al. showed that calcitriol significantly reduced tubulo-interstitial inflammation in a rodent renal injury model [6]. The authors linked this renoprotective effect to calcitriol-induced up-

\footnotetext{
${ }^{1}$ ICU Department, Universitair Ziekenhuis Brussels, Vrije Universiteit Brussel, 101 Laarbeeklaan, 1090 Brussels, Jette, Belgium

${ }^{2}$ To whom correspondence should be addressed at ICU Department, Universitair Ziekenhuis Brussels, Vrije Universiteit Brussel, 101 Laarbeeklaan, 1090 Brussels, Jette, Belgium. E-mail: Patrick.Honore@az.vub.ac.be
}

regulation of zinc finger protein $\mathrm{A} 20$, a de-ubiquitinating enzyme which causes disruption of nuclear factor kappa B (NF- $\mathrm{B}$ B) dependent intracellular chemo- and cytokine production and suppresses apoptotic pathways [7].

Whether this novel compelling insight into the cellular mechanism of action of vit D may change the current or future therapeutic approach of CKD is uncertain. Factors contributing to the progression of CKD are indeed not limited to perturbed mineral metabolism, chronic inflammation, and oxidative stress but also include proteinenergy wasting, pre-existing heart failure, arterial hypertension, iron deficiency, and dialysis-related injury [8]. Patients with CKD often present a "leaky gut" and/or profound alterations in gut microbial flora. Increased gut wall permeability promotes translocation of bacteria and endotoxin which continuously fuels an inflammatory state. Changes in the intestinal microbiome are highly determined by intraluminal influx of urea, dietary constituents, and occasional antimicrobial therapy $[8,9]$. Of note is that both intestinal leakage and microbial environment show large interpatient variability and cannot be quantified. In addition, accumulation of protein-bound toxins (e.g., pcresyl sulfate and indoxyl sulfate) [10] and altered mucosal defense mechanisms may also contribute to cardiovascular events and sustain inflammation.

Despite robust in vitro arguments to support the renoprotective potential of vit $\mathrm{D}$ supplementation in CKD, hard clinical outcome data are lacking. A metaanalysis of randomized controlled trials showed that treatment with paricalcitol, a selective activator of the vit $\mathrm{D}$ receptor promoting sequestration of $\mathrm{NF}-\mathrm{\kappa B}$ signaling [11], lowered the risk of cardiovascular events in CKD patients but failed to reduce proteinuria and to protect renal function [12]. Vit D therapy neither improved vascular endothelial function nor attenuated inflammation in patients with CKD treated either with cholecalciferol or calcitriol for 6 months [13]. Likely explanations of these negative outcomes are 
the use of different vit $\mathrm{D}$ dose regimens, attempted correlations with 25OHD instead of active hormone plasma levels, the effect of renal replacement therapy, and the possible impact of other pathophysiological pathways that are related with vit $\mathrm{D}$. Among the latter, the reninangiotensin-aldosterone system (RAAS) stands out as a crucial regulator of intravascular volume and blood pressure in CKD. Calcitriol modulates the RAAS system by suppressing renin gene and angiotensin-converting enzyme expression [14, 15]. Blocking RAAS with angiotensin-converting enzyme inhibitors or angiotensin receptor blockers controls arterial hypertension, reduces proteinuria, and prevents or reverses endothelial dysfunction and atherosclerosis in patients with CKD [16]. Endothelin (ET)-1-induced signaling pathways in vascular smooth muscle cells represent another attractive target for improving cardiovascular morbidity in CKD. ET type A receptor blockade reduced vascular inflammation and smooth muscle cell differentiation in rats with CKD [17]. In experimental CKD models, the combination of RAAS inhibition with ET receptor antagonism ameliorated proteinuria, renal structural changes, and molecular markers of glomerulosclerosis, renal fibrosis, or inflammation more effectively than RAAS inhibitors or ET receptor antagonists alone [18].

Taken together, the findings of Zhao et al. confirm the immune-inflammatory modulating capacity of vit $\mathrm{D}$ and expand the molecular basis to further explore its therapeutic potential in CKD. However, whether unveiling the role of A20 in calcitriol's renoprotection will lead to more effective treatment remains to be proven.

Authors' Contributions PMH and HDS designed and wrote the paper. Both authors agree with the last version.

\section{COMPLIANCE WITH ETHICAL STANDARDS}

Conflict of Interest. The authors declare that they have no competing interests.

\section{REFERENCES}

1. Bikle, D.D. 2014. Vitamin D metabolism, mechanism of action, and clinical applications. Chemistry \& Biology 21 (3): 319-329. https:// doi.org/10.1016/j.chembiol.2013.12.016.
2. Colotta, F., B. Jansson, and F. Bonelli. 2017. Modulation of inflammatory and immune responses by vitamin D. Journal of Autoimmunity S0896-8411 (17): 30463-30468. https://doi.org/10.1016/ j.jaut.2017.07.007.

3. Yadav, A.K., V. Kumar, V. Kumar, D. Banerjee, K.L. Gupta, and V. Jha. 2017. The effect of vitamin D supplementation on bone metabolic markers in chronic kidney disease. Journal of Bone and Mineral Research. https://doi.org/10.1002/jbmr.3314.

4. Akchurin, O.M., and F. Kaskel. 2015. Update on inflammation in chronic kidney disease. Blood Purification 39 (1-3): 84-92. https:// doi.org/10.1159/000368940.

5. Wolf, M., A. Shah, O. Gutierrez, E. Ankers, M. Monroy, H. Tamez, D. Steele, Y. Chang, C.A. Camargo Jr., M. Tonelli, and R. Thadhani. 2007. Vitamin D levels and early mortality among incident hemodialysis patients. Kidney International 72 (8): 1004-1013.

6. Zhao H, Xia Y, Gan H.2017. Calcitriol ameliorates angiotensinIIinduced renal injury partly via upregulating A20. Inflammation. Dec;40:1884-1893. https://doi.org/10.1007/s10753-017-0629-y.

7. Evans, P.C., H. Ovaa, M. Hamon, P.J. Kilshaw, S. Hamm, S. Bauer, H.L. Ploegh, and T.S. Smith. 2004. Zinc-finger protein A20, a regulator of inflammation and cell survival, has de-ubiquitinating activity. The Biochemical Journal 378 (Pt 3): 727-734.

8. Lau, W.L., K. Kalantar-Zadeh, and N.D. Vaziri. 2015. The gut as a source of inflammation in chronic kidney disease. Nephron 130 (2): 92-98. https://doi.org/10.1159/000381990.

9. Lau, W.L., and N.D. Vaziri. 2017. The leaky gut and altered microbiome in chronic kidney disease. Journal of Renal Nutrition 27: 458-461. https://doi.org/10.1053/j.jrn.2017.02.010.

10. Honore, P.M., R. Jacobs, E. De Waele, V. Van Gorp, J. De Regt, O. Joannes-Boyau, W. Boer, and H.D. Spapen. 2015. A fresh look into the pathophysiology of ischemia-induced complications in patients with chronic kidney disease undergoing hemodialysis. International Journal of Nephrology and Renovascular Disease 8: 25-28.

11. Tan, X., X. Wen, and Y. Liu. 2008. Paricalcitol inhibits renal inflammation by promoting vitamin D receptor-mediated sequestration of NF-kappaB signaling. Journal of the American Society of Nephrology 19: 1741-1752. https://doi.org/10.1681/ASN.2007060666.

12. Hu, X., J. Shang, W. Yuan, S. Zhang, Y. Jiang, B. Zhao, Y. Duan, J. Xiao, and Z. Zhao. 2017. Effects of paricalcitol on cardiovascular outcomes and renal function in patients with chronic kidney disease: a meta-analysis. Herz. https://doi.org/10.1007/s00059-017-4605-y.

13. Kendrick, J., E. Andrews, Z. You, K. Moreau, K.L. Nowak, H. Farmer-Bailey, D.R. Seals, and M. Chonchol. 2017. Cholecalciferol, calcitriol, and vascular function in CKD: a randomized, doubleblind trial. Clinical Journal of the American Society of Nephrology 12 (9): 1438-1446.

14. Yuan, W., W. Pan, J. Kon, W. Zheng, F.L. Szeto, et al. 2007. 1,25Dihydroxyvitamin D3 suppresses renin gene transcription by blocking the activity of the cyclic AMP response element in the renin gene promoter. Journal of Biological Chemistry 282 (41): 29821-29830.

15. Lin, M., P. Gao, T. Zhao, Lei He, M. Li, et al. 2016. Calcitriol regulates angiotensin-converting enzyme and angiotensin converting-enzyme 2 in diabetic kidney disease. Molecular Biology Reports 43 (5): 397-406.

16. Werner, C., J. Pöss, and M. Böhm. 2010. Optimal antagonism of the renin-angiotensin-aldosterone system: do we need dual or triple therapy? Drugs 70 (10): 1215-1230. https://doi.org/10.2165/ 11537910-000000000-00000 Review.

17. Larivière, R., A. Gauthier-Bastien, R.V. Ung, J. St-Hilaire, F. MacWay, D.E. Richard, and M. Agharazii. 2017. Endothelin type a receptor blockade reduces vascular calcification and inflammation 
in rats with chronic kidney disease. Journal of Hypertension 35: 376-384.

18. Komers, R., and H. Plotkin. 2016. Dual inhibition of reninangiotensin-aldosterone system and endothelin-1 in treatment of chronic kidney disease. American Journal of Physiology. Regulatory, Integrative and Comparative Physiology 310: R877-R884. https://doi.org/10.1152/ajpregu.00425.2015. 